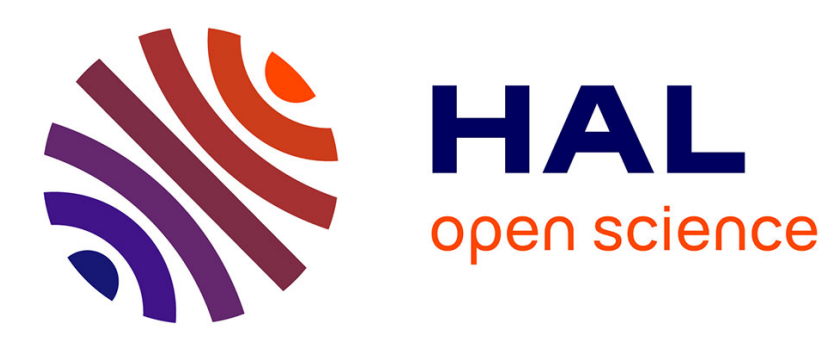

\title{
Comment comparer l'ingestibilité en vert des variétés de graminées? Premiers résultats
}

\author{
Michel Gillet, Claude Noel, Rémy Traineau
}

\section{To cite this version:}

Michel Gillet, Claude Noel, Rémy Traineau. Comment comparer l'ingestibilité en vert des variétés de graminées? Premiers résultats. Agronomie, 1983, 3 (9), pp.867-872. hal-00884582

\section{HAL Id: hal-00884582 \\ https://hal.science/hal-00884582}

Submitted on 1 Jan 1983

HAL is a multi-disciplinary open access archive for the deposit and dissemination of scientific research documents, whether they are published or not. The documents may come from teaching and research institutions in France or abroad, or from public or private research centers.
L'archive ouverte pluridisciplinaire HAL, est destinée au dépôt et à la diffusion de documents scientifiques de niveau recherche, publiés ou non, émanant des établissements d'enseignement et de recherche français ou étrangers, des laboratoires publics ou privés. 


\title{
Comment comparer l'ingestibilité en vert des variétés de graminées? Premiers résultats
}

\author{
Michel GILLET \& Claude NOEL \\ avec la collaboration technique de Rémy TRAINEAU \\ I.N.R.A., Station d'Amélioration des Plantes fourragères, F 86600 Lusignan.
} sélectionneurs de graminées sont obligés de donner le choix aux animaux. Nous avons monté une expérience pour étudier, sur 6 génotypes, les corrélations entre, d'une part, l'appétibilité mesurée avec des moutons en cafétéria d'auges, d'autre part, l'ingestibilité et la digestibilité mesurées avec des moutons en cage. L'étude a porté sur 1 brome, 2 ray-grass d'Italie et 3 fétuques élevées. Deux des fétuques ont été également étudiées à l'aide de chèvres laitières.

Cette expérimentation a donné des résultats inattendus :

- aucune corrélation n'est apparue entre l'ingestibilité et les deux autres critères : digestibilité (mesurée avec les mêmes moutons) et appétibilité. Ceci reste vrai, même lorsque des différences significatives existent entre variétés pour ces trois critères.

- l'appétibilité et la digestibilité (mesurées avec des moutons différents) ont généralement été liées entre elles.

- le classement de deux fétuques pour la production de lait de chèvres a été le même que leur classement pour l'appétibilité et la digestibilité avec des moutons.

- le classement entre espèces obtenu avec la cafétéria a été le même que celui généralement indiqué par les agriculteurs : brome $>$ ray-grass $>$ fétuque élevée. L'ingestibilité, mesurée en cages, a donné un classement différent : ray-grass $\geqslant$ fétuque $=$ brome.

Une question se pose alors : pour déterminer les quantités ingérées par des animaux en conditions agricoles, le problème des préférences ne jouerait-il pas un rôle plus important qu'on ne le croit généralement et, en tout cas, plus important que dans le cas de moutons en cages?

Mots clés additionnels : Appétibilité, valeur alimentaire, digestibilité, cafétéria, sélection.

In order to check a sufficiently large number of genotypes for their consumability, grass breeders cannot avoid giving free choice to the animals. We have carried out an experiment to study, on 6 genotypes, the correlations between palatability as measured with sheep in a trough cafeteria on one hand, and intake and digestibility as measured with sheep in crates on the other hand. The experiment was carried out on one bromus, two rye grasses and three tall fescues. Two of the fescues were also studied with dairy goats. We have obtained the following results :

1) no correlation appeared between intake and the other two criteria : digestibility (measured with the same sheep) and palatability. This was true, even when significant differences between varieties came out for the three criteria.

2) palatability and digestibility (measured with different sheep) were correlated.

3) the ranking of the two fescues for goat milk production was the same as their ranking for palatability and digestibility for sheep.

4) the three species ranked with the cafeteria in the same way as generally indicated by farmers: bromus $>$ rye grass $>$ fescue. Intake in crates ranked differently : rye grass $\geqslant$ fescue $=$ bromus.

The question thus arises : in determining intake by animals under farm conditions, might the problem of palatability not be more important than usually thought, at least more important that it is for sheep in crates ?

Additional key words : Palatability, feeding value, digestibility, cafeteria, plant breeding. 


\section{INTRODUCTION}

Habituellement, les mesures d'ingestibilité des fourrages servent à calculer des rations. L'essentiel n'est donc pas que les valeurs obtenues soient précises, mais qu'elles soient directement applicables à la réalité agricole, c'est-à-dire qu'elles ne soient pas biaisées par la méthode.

Pour des raisons matérielles évidentes, il n'est pas possible de mesurer l'ingestibilité de tous les fourrages avec tous les types d'animaux utilisateurs et dans tous les types de situation (pâture, affouragement, conserve). Aussi, les zootechniciens ont-ils mis au point un test unique, sur béliers castrés, à l'auge, en cages.

Mais les sélectionneurs, lorsqu'ils veulent améliorer l'ingestibilité d'un fourrage, ne trouvent pas dans les tests dont nous venons de parler, l'instrument dont ils ont besoin. Les génotypes qu'ils doivent étudier, dans des conditions de culture semblables, sont trop nombreux. Il est secondaire que la valeur absolue des chiffres obtenus soit biaisée ou non par la méthode, pourvu que les classements des génotypes soient corrects.

Pour résoudre le problème posé par les grands nombres de génotypes à tester, la seule solution consiste à donner le choix aux animaux. Les sélectionneurs ont donc mis au point, depuis lor.gtemps, de tels tests, appelés "cafétéria " (Petersen et al., 1958; Buckner \& Burrus, 1962; GILlET \& JADAS-HECART, 1965 ; GILLET et al., 1983).

Il existe plusieurs techniques de cafétéria et elles donnent des classements parallèles (GILLET et al., 1983).

Naturellement, si l'animal a le choix, on ne mesure pas exactement l'aptitude du fourrage à être ingéré en grande quantité, mais son aptitude à exciter l'appétence de l'animal. Cette aptitude s'appelle appétibilité. Plus concrètement, nous appellerons ici appétibilité le résultat des tests en cafétéria.

Cette appétibilité devrait logiquement être l'un des éléments de l'ingestibilité, mais quel est le degré de liaison entre ces deux mesures? Pour le savoir, nous avons comparé, en 1979, 6 fourrages de graminées pures, en cafétéria d'auges et avec des moutons en cage. Ces derniers nous ont permis d'étendre la comparaison à la digestibilité.

Par la même occasion, nous avons étudié l'ingestibilité de certains de ces fourrages, en parallèle, avec des chèvres en lactation, qui vivaient en lots, en stabulation.

Nous avons obtenu des résultats inattendus, que nous présentons ici. Ces résultats nous amènent à poser une question : quel est le meilleur test permettant de classer les ingestibilités en vert de différentes variétés de graminées?

\section{MATÉRIEL ET MĖTHODES}

\section{A. Matériel végétal}

Six fourrages ont été comparés pour l'appétibilité, l'ingestibilité et la digestibilité avec moutons : 2 ray-grass d'Italie que nous appellerons $\mathrm{R} 1$ et $\mathrm{R} 2$, un brome $\mathrm{B} 1$ et 3 fétuques élevées $\mathrm{F} 1, \mathrm{~F} 2, \mathrm{~F} 3$.

Deux de ces fétuques, F1 et F2, ont été également comparées à l'aide de chèvres laitières, ainsi qu'un ray-grass d'Italie, R3, différent des 2 variétés déjà mentionnées.

Les rythmes de développement de tous ces fourrages sont très voisins (épiaisons groupées en $4-5 \mathrm{j}$ ). Ils avaient été semés au cours d'années différentes, sur des emplacements différents. Pour cette raison, nous n'indiquons pas les noms des variétés, le terrain ou l'âge de la culture pouvant éventuellement modifier les classements. Seule, la comparaison des méthodes nous intéresse ici.

La fumure azotée a été de 60 unités/ha en novembre, 120 en février.

Les mesures ont été effectuées tout au long de la $1^{\text {re }}$ pousse de l'herbe du 23 avril au 25 mai, soit 5 semaines, en 1979.

\section{B. Ingestibilité et digestibilité sur moutons en cages}

Pour chaque variété à tester, on a utilisé 6 moutons mâles adultes, castrés, de race Texel: 5 maintenus en cage à digestibilité et 1 autre en cage au sol pour la seule mesure de la quantité ingérée, que l'on sait plus variable que la digestibilité. Les lots d'animaux ont été constitués en fonction de leur âge et de leur poids. Celui-ci est passé de $58,8 \mathrm{~kg}$ en moyenne en début d'expérience, à $63,1 \mathrm{~kg}$ à la fin.

Le fourrage était coupé chaque jour en fin de matinée, puis haché en brins de 3 à $5 \mathrm{~cm}$ et stocké à même le sol dans un local ; il était distribué ad libitum, permettant un refus d'environ 10 p. 100 , au repas du soir et à celui du lendemain matin, sauf en début de semaine où il était distribué aux 2 repas du lundi et à celui du mardi matin.

Les mesures ont eu lieu chaque semaine pendant $5 \mathrm{j}$. Les résultats sont présentés ici sous forme de moyennes hebdomadaires. Ils sont exprimés en pourcentage dans le cas de la digestibilité de la matière organique, et en $g$ de matière sèche par $\mathrm{kg}$ de poids métabolique pour l'ingestibilité. Une analyse de covariance de l'ingestibilité sur le poids métabolique initial de chaque animal a été tentée mais n'a pas amélioré la précision.

\section{Appétibilité en cafétéria d'auges}

Nous avons utilisé le dispositif décrit par GILlET et al. (1983).

Dans chaque auge, on apportait $2 \mathrm{~kg}$ d'herbe verte : celle qui venait d'être récoltée et hachée le matin pour les moutons en cage. La distribution se faisait en fin de matinée.

Nous avons ainsi effectué 4 cafétérias par semaine. Entre temps, les moutons séjournaient sur un paddock, sur lequel on apportait chaque jour un tas assez gros de chacune des variétés récoltées: ainsi, ils avaient le même choix à tout moment.

Les résultats analysés par jour se sont montrés très semblables d'un jour à l'autre. Ce sont donc des moyennes par semaine qui sont présentées et analysées ici. Elles sont exprimées en grammes de matière sèche consommée par auge et par jour.

\section{Ingestibilité et production laitière sur chèvres}

Pour chaque variété testée, on a utilisé 8 chèvres de race alpine chamoisée. Le poids moyen des animaux est passé de $56,6 \mathrm{~kg}$ en début d'expérience à 57,6 à la fin. Les lots avaient été constitués d'après le numéro de lactation, l'âge, la production de lait et le poids des animaux ainsi que d'après l'évolution de ces deux grandeurs pendant 6 semaines après les mises bas, enfin d'après les capacités individuelles d'ingestion durant une période pré-expérimentale de 2 semaines, pendant laquelle les animaux avaient reçu de l'ensilage de maïs.

Les chèvres vivaient par lots, étant seulement bloquées au cornadis, lors de 4 repas journaliers d'une heure chacun.

Les fourrages étaient coupés chaque jour en début 
d'après-midi et distribués le soir et le lendemain matin, de façon à permettre un refus d'environ 15 p. 100 en début d'expérience (herbe feuillue) à 30 p. 100 en fin d'expérience (herbe épiée). Ce taux élevé est habituel pour la chèvre, animal réputé «difficile», pour permettre des niveaux de production normaux. L'herbe n'était pas hachée car le hachage nuit à la consommation chez cet animal. En outre, $600 \mathrm{~g}$ d'orge étaient distribués par jour plus $100 \mathrm{~g}$ par $1 / 2 \mathrm{~kg}$ de lait produit au-dessus de $3,5 \mathrm{~kg}$.

Les mesures ont eu lieu chaque semaine pendant 4 jours. Les résultats sont présentés ici sous forme de moyennes hebdomadaires, après prise en compte, par l'analyse de la covariance, des caractéristiques initiales des animaux lorsque cela a pu améliorer la précision. L'ingestibilité est exprimée en $\mathrm{g}$ de matière sèche par $\mathrm{kg}$ de poids métabolique, la production laitière en litres par jour.

\section{RESULTATS}

La figure 1 représente les résultats obtenus avec les moutons : appétibilité en cafétéria, ingestibilité et digestibilité en cages.

Comme on pouvait s'y attendre, les caractéristiques mesurées en cages vont diminuant quand la plante vieillit : cette diminution est régulière pour la digestibilité, elle ne commence qu'en milieu de pousse pour l'ingestibilité. Cela crée naturellement une corrélation entre ces deux grandeurs, corrélation bien connue (JARRIGE, 1978) mais là n'est pas notre propos.

L'appétibilité, elle, n'évolue pas, en moyenne, avec le temps. Cela tient à la méthode : par définition, on arrête l'expérience lorsque la moitié environ de l'herbe offerte a été consommée.

Ce qui nous intéresse ici c'est le parallélisme des classements entre les diverses méthodes. A première vue, l'étude de la quantité ingérée amène à un classement des variétés différent de celui obtenu par les deux autres méthodes. Ces dernières, qui évaluent l'appétibilité et la digestibilité, permettent des classements plus comparables entre eux.

Cette impression est confirmée par le calcul des corrélations entre les 3 tests. Pour éliminer le facteur de variation «stade de la plante», cette corrélation a été calculée séparément chaque semaine et, sur les moyennes variétales, pour l'ensemble de la période. Elles sont indiquées figure 2. Avec 4 degrés de liberté ( 6 variétés), la valeur limite de $\mathrm{r}$ au seuil 0,05 était de 0,81 .

L'appétibilité, mesurée dans l'espoir d'avoir une idée de l'ingestibilité, s'avère en fait liée non pas à celle-ci mais à la digestibilité. Cela ne s'explique ni par la proportion de feuilles, mesurée 4 semaines sur les 5 , ni par les teneurs en eau ou en azote, mesurées chaque semaine : ces caractères se classent de façon tout à fait différente.

On peut se demander si cette corrélation entre appétibilité et digestibilité n'est pas due uniquement à l'existence de 3 espèces différentes de graminées dans l'expérience, ou si elle est valable également entre génotypes d'une même espèce. La figure 3 représente les points correspondants.

Figure 1

Résultats obtenus sur moutons.

(Des chiffres apparemment négatifs s'expliquent par le fait que la matière sèche n'est pas déterminée sur le même échantillon pour le distribué et pour les refus.)

Results obtained with sheep.

(Apparently negative figures are due to the fact that dry matter determination was not worked out on the same sample for distributed and for refused grass.)
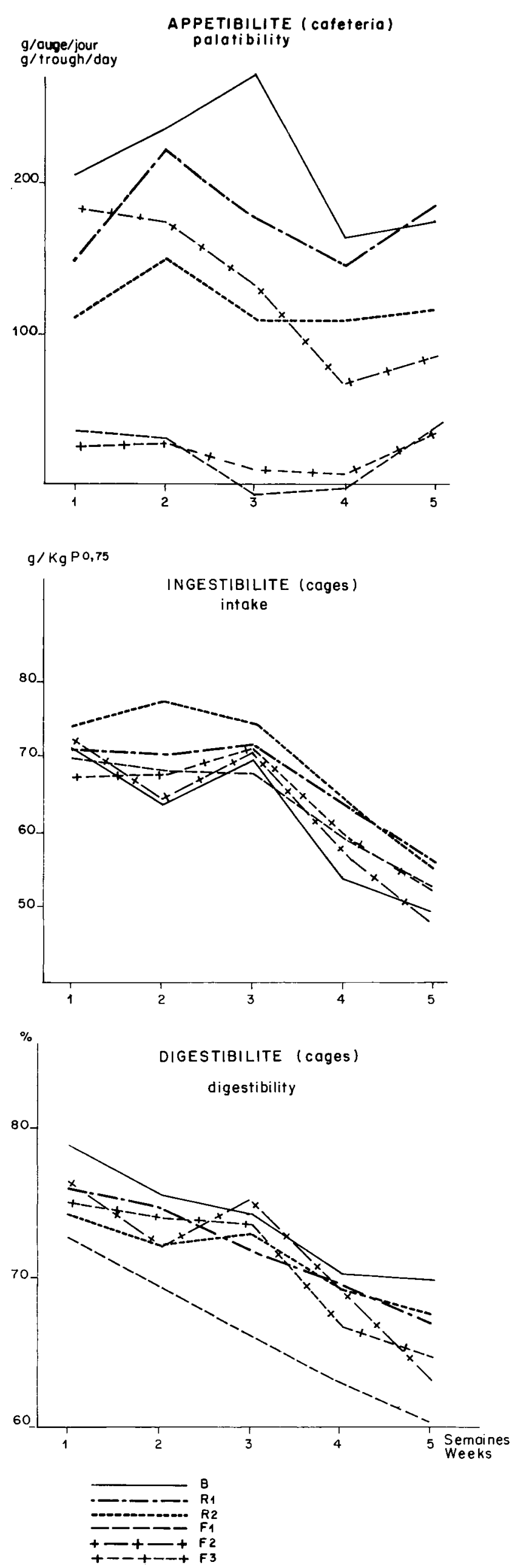


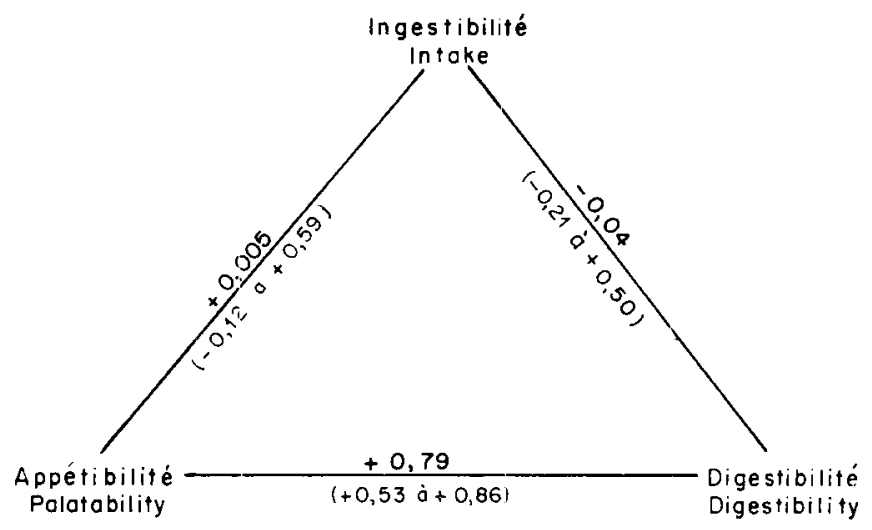

Figurc 2

Corrélations entre appétibilité, ingestibilité et digestibitité.

En gras: sur les moyennes de la période.

Entre parenthèses: semaine par semaine.

Correlations between palatability, intake and digestibility.

Heavy type: between means over the period.

In brackets : week per week.

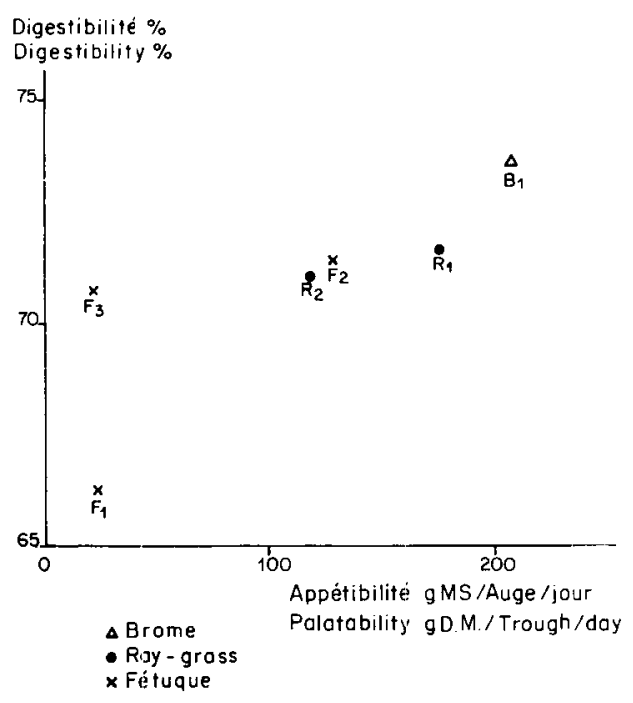

Figure 3

Analyse de la corrélation entre appétibilité et digestibilité. Analysis of the correlation between palatability and digestibility.

On voit que le point représentant la fétuque $\mathrm{F} 3$ empêche, à lui seul, de répondre à la question. Cette fétuque, cultivée dans une parcelle très éloignée de toutes les autres, sur un terrain plus fertile, y était beaucoup plus vigoureuse. Or, lorsqu'on mesure l'appétibilité en pâture, on constate que les plantes les moins vigoureuses sont préférées, cette relation restant vraie à l'auge (GILLET et al., 1983). Ajoutons que les 3 fétuques $F 1, F 2$ et $F 3$, cultivées ensemble dans les essais blocs, ont souvent été comparées entre elles pour l'appétibilité, dans notre station. Régulièrement, F3 est égale ou supérieure à F2, F1 étant toujours la moins appréciée. On peut donc penser que dans l'expérience présente, l'appétibilité de $\mathrm{F} 3$ a été sérieusement sousestimée du seul fait de la différence de parcelle. Le point correspondant sur la figure 3 se trouverait alors trop à gauche. Si on l'imagine à sa place "normale ", près de F2, alors la corrélation entre appétibilité et digestibilité semble s'appliquer aussi bien entre variétés d'une même espèce qu'entre espèces.
Pourquoi les liaisons observées entre l'ingestibilité et les deux autres caractères (fig. 2) sont-elles vraiment nulles ? Les différences d'ingestibilité pouvant exister entre génotypes de digestibilité égale (DEMARQUILLY \& WeISS, 1970) ne nous semblent pas une raison suffisante. Pour comprendre, nous avons d'abord examiné le degré de précision des 3 tests. Une corrélation ne peut en effet apparaitre que si les variables corrélées sont mesurées avec un minimum de précision.

Le test le plus précis a été de loin celui d'appétibilité : la valeur du $\mathrm{F}$ de Fisher correspondant à l'effet « variétés » est de 822,5 sur les moyennes et, dans le cas des analyses hebdomadaires, varie entre 103,7 et 252,8 (la limite au seuil de 1 p. 100 étant de 3,5 et 4,1 respectivement).

En ce qui concerne le test de digestibilité, le F est de 24,2 pour les moyęnnes et varie entre 4,8 et 14,3 dans le cas des analyses hebdomadaires (la limite au seuil de 1 p. 100 étant respectivement de 3,2 et 4,0$)$.

Le test d'ingestibilité a été le moins précis, avec un $\mathrm{F}$ d'ensemble égal à $5,4(\lim .1 \mathrm{p} .100=3,20)$ et des $\mathrm{F}$ hebdomadaires variant, 4 semaines sur 5 , entre 0,5 et 1,9 , celui de la semaine $\mathrm{n}^{\circ} 2$ étant de $3,7(\mathrm{lim} .5 \mathrm{p} .100=2,7$; lim. 1 p. $100=4,0)$. On sait que le test d'ingestibilité n'est jamais précis, compte tenu de la forte variation entre animaux. Nous n'avons pas pu l'améliorer par l'analyse de covariance sur le poids métabolique initial des animaux, car la corrélation avec les ingestions ultérieures, pour les moutons d'un même lot, s'est avérée nulle. Néanmoins, on peut dire que, par rapport à la précision habituelle des tests d'ingestibilité, nos résultats de 1979 n'ont pas été mauvais.

Cette faible précision de l'ingestibilité explique-t-elle l'absence de corrélation avec l'appétibilité ou la digestibilité ? Non, puisque les différences variétales d'ingestibilité sont quand même significatives au seuil de 1 p. 100 sur l'ensemble de la période. Si par ailleurs on examine les résultats de la semaine 2 , où les différences variétales d'ingestibilité sont également significatives (au seuil 5 p. 100), les corrélations ne sont pas meilleures $(-0,12$ avec l'appétibilité, $-0,21$ avec la digestibilité).

Examinons maintenant les résultats obtenus à l'aide des chèvres.

L'ingestibilité des 3 variétés testées avec ces animaux n'a pas pu être corrigée par l'analyse de covariance sur leur capacité initiale d'ingestion lorsqu'ils mangeaient du maïs fourrage : la corrélation intra-lots avec l'ingestion ultérieure d'herbe verte s'est avérée nulle. Par contre, le poids vif initial des chèvres est apparu lié, dans un même lot, à leur consommation ultérieure $(\mathrm{r}=0,58$, limite au seuil 1 p. $100=0,27$ ). Cela a permis, grâce à l'analyse de covariance, de corriger les données. Le résultat a été que les 3 fourrages ont été ingérés pratiquement de la même manière $(F=0,27)$. Ce phénomène tient sans doute au fait qu'on a autorisé des refus très importants. Les animaux, ayant eu la possibilité de trier les organes, ont pu manger autant de chaque fourrage, mais peut-être une proportion variable des divers organes de la plante.

Avec les chèvres, nous avons mesuré également la production laitière et nous avons pu la corriger par l'analyse de covariance sur la production laitière initiale (corrélation intra-lots : $r=0,80$ ). Les résultats sont représentés par la figure 4 . La valeur du $F$ pour les différences variétales est de 23,3 , donc très hautement significative ( $\mathrm{F}$ lim. 1 p. $100=4,8$ ). On peut remarquer que les 2 fétuques $F 2$ et F1 se classent, pour l'efficacité laitière avec chèvres, comme elles se classent pour l'appétibilité et pour la digestibilité, alors qu'elles sont inversées en ingestibilité par les moutons en cage. En ce qui concerne le ray-grass R3, il est plus 


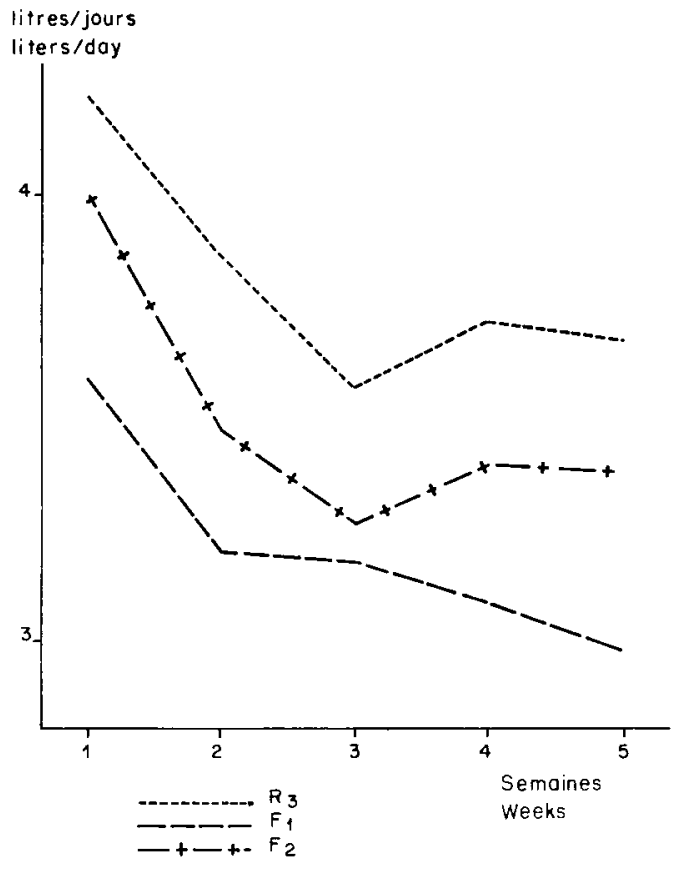

Figure 4

Production de lait de chèvres (données corrigées par l'analyse de covariance sur la production de lait initiale.)

Goat milk production (as corrected through covariance analysis on initial milk production.)

difficile de conclure puisqu'il ne figurait pas dans les autres tests. On peut seulement dire qu'il est supérieur aux 2 fétuques, comme le sont les 2 ray-grass en ingestibilité et R1 en appétibilité.

\section{DISCUSSION}

Nous obtenons les résultats suivants :

- la réponse à la principale question que nous nous posions, est négative : l'appétibilité n'est absolument pas liée à l'ingestibilité. Ceci devrait nous inciter à rejeter la cafétéria.

- mais l'appétibilité montre une bonne liaison, à laquelle il était difficile de s'attendre, avec la digestibilité : c'est une raison pour conserver la cafétéria.

- l'ingestibilité n'est absolument pas liée à la digestibilité, mesurée pourtant à l'aide des mêmes moutons. La production de lait de chèvres donne, pour 2 fétuques, un classement qui n'est pas celui de leur ingestibilité, mais celui de leur appétibilité et de leur digestibilité. Enfin, dans notre expérience, le brome, qui était cultivé à côté des ray-grass, s'est classé premier pour l'appétibilité, dernier pour l'ingestibilité. Or, tous les agriculteurs ayant cultivé ce brome témoignent que les vaches laitières, au pâturage, le préfèrent nettement aux ray-grass (et à fortiori aux fétuques élevées).

Nos résultats nous amènent donc à poser la question suivante: les préférences gustatives ne seraient-elles pas importantes dans le déterminisme des quantités ingérées en conditions agricoles? ou peut-être seulement pour les vaches laitières au pâturage? Des animaux maintenus en cages vivent dans des conditions différentes de la pratique : ils ne sont pas en groupe et n'ont que des besoins d'entretien. L'affouragement à l'auge n'est pas la pâture.

Pourtant, des comparaisons entre ingestibilité par moutons en cages et ingestibilité par vaches laitières ont montré une bonne corrélation, si cette ingestibilité était inférieure à $80 \mathrm{~g} / \mathrm{kg}$ de poids métabolique. Ces vaches étaient à l'auge, en stabulation entravée (DEMARQuilly \& WeISS, 1971). Cette corrélation a été encore significative, quoique moins bonne, entre moutons en cages et bœufs à l'engrais en stabulation libre (ibid.). Pour l'ingestibilité par des chèvres et des brebis, on sait seulement qu'on retrouve bien des facteurs de variation que l'on sait importants avec des moutons en cage (âge de la plante, vitesse de digestion, teneur en matière sèche). Mais dans toutes ces études, on comparait des échantillons d'une même espèce de graminée, associée à un trèfle blanc, et qui différaient par de nombreux facteurs de variation non isolés les uns des autres (numéro de la pousse, stade de végétation, fertilisation, rarement variété). Or, la valeur et même le signe d'une corrélation entre deux grandeurs dépend du facteur qui les fait varier. Si plusieurs facteurs interviennent, ceux qui entraînent les plus fortes variations des caractères étudiés sont souvent déterminants pour la corrélation. Dans le cas présent, on sait que le stade de végétation a un tel effet majeur. On peut donc craindre que les éventuelles corrélations liées aux différences génétiques, n’aient pas pu apparaître.

Il ne semble pas qu'il y ait de données sur l'ingestibilité en pâture, compte tenu de la difficulté de la mesurer.

\section{CONCLUSION}

Nous avions monté cette expérience pour voir si l'appétibilité en cafétéria permettait de classer correctement les génotypes de graminées pour leur ingestibilité en vert. A notre connaissance, c'est la première expérience de ce genre.

Nous n'avons pas obtenu de réponse claire à la question ainsi posée, car nous avons été amenés à nous demander quelle serait la meilleure méthode pour comparer les ingestibilités en vert des graminées: les préférences des animaux ne joueraient-elles pas un rôle important dans le déterminisme des quantités ingérées, en conditions agricoles?

Par ailleurs, nous avons mis en évidence une corrélation entre appétibilité et digestibilité.

Etant donné l'importance de ces conclusions, il était utile de renouveler cette expérience. C'est ce que nous avons réalisé depuis plusieurs années. Les résultats, en cours de dépouillement, seront publiés dès que possible.

Reçu le 17 novembre 1981 Accepté le 7 mai 1983.

\section{REMERCIEMENTS}

Nous remercions M. Lionel Huguet, Chef du Laboratoire de Production et Utilisation des Fourrages, pour les moyens de travail qu'il a mis à notre disposition et pour les conseils qu'il nous a prodigués. 


\section{RÉFÉRENCES BIBLIOGRAPHIQUES}

Buckner R. C., Burrus P. B., 1962. Comparison of techniques for evaluating palatability differences among tall fescue strains. Crop Sci., 2 (1), 55-57.

Demarquilly C., Weiss Ph., 1970. Tableaux de la valeur alimentaire des fourrages. S.E.I., Etude $\mathrm{n}^{\circ} 42,64 \mathrm{p}$.

Demarquilly C., Weiss Ph., 1971. Liaisons entre les quantités de matière sèche de fourrages verts ingérées par les moutons et celles ingérées par les bovins. Ann. Zootech., 20 (2), 119-134.

Gillet M., Jadas-Hecart J., 1965. La flexibilité des feuilles, critère de sélection de la fétuque élevée en tant que facteur d'appétibilité. Fourrages, 22, 6-10.
Gillet M., Noël C., Jadas-Hecart J., 1983. La cafétéria d'auges, méthode d'étude de l'appétibilité des fourrages. Agronomie, 3 (8), 817-821.

Jarrige R., 1978. Consommation d'aliments et d'eau, in Alimentation des ruminants. Ed. INRA, p. 177-206.

Petersen R. G., Weswig P. H., Cowen J. R., 1958. Measuring palatability differences in tall fescue by grazing sheep. Agron. J., 50 (3), 117-119. 